\title{
Distribución, densidad y nicho isotópico en reptiles y mamíferos del desierto absoluto de Atacama; con registro de saurofagia entre reptiles
}

\author{
Distribution, density and isotopic niche of reptiles and mammals from the Atacama absolute \\ desert; with record of saurophagy among reptiles
}

\author{
Gabriel Lobos ${ }^{1,2,3, *}$, Gianina Tapia ${ }^{2}$, Alejandra Alzamora ${ }^{1,2}$ \& Osvaldo Rojas ${ }^{3}$ \\ ${ }^{1}$ Centro de Gestión Ambiental y Biodiversidad, Facultad de Ciencias Veterinarias y Pecuarias, Universidad de Chile, Santiago, Chile. \\ 2Ecodiversidad Consultores, Santiago, Chile. \\ ${ }^{3}$ Museo de Historia Natural y Cultural del Desierto de Atacama, Calama, Chile. \\ E-mail: galobos@ug.uchile.cl
}

\section{RESUMEN}

El desierto absoluto de Atacama, carente de vegetación, se extiende por 740 kilómetros de latitud en Chile. En este ecosistema, la presencia de vertebrados de baja movilidad hace suponer el desarrollo de capacidades adaptativas especiales y la presencia de interrelaciones ecosistémicas que han sido poco exploradas. En este contexto, realizamos un levantamiento de información en dos gradientes de $76 \mathrm{~km}$ cada una, donde estudiamos la distribución y densidad de reptiles y mamíferos. Por otra parte, utilizamos isotopos estables para explorar las relaciones tróficas de los reptiles que habitan en este ecosistema. Observamos que las poblaciones de reptiles y mamíferos son poco frecuentes y presentan bajas densidades. Del punto de vista trófico, los pececillos de plata pertenecientes al orden Zygentoma son una presa clave en la comunidad de reptiles, los que se sobreponen en el consumo de fuentes tróficas, pero difieren en su posición en la cadena trófica. La información es complementada con antecedentes de predación entre reptiles de este ecosistema extremo.

Palabras clave: desierto absoluto, mamíferos, nicho isotópico, reptiles, saurofagia.

\section{ABSTRACT}

The hyperarid Atacama Desert, without vegetation, extends for $740 \mathrm{~km} \mathrm{~N}$ to $\mathrm{S}$ in Chile. The presence of vertebrates with low mobility in this environment indicates the development of special adaptive capacities and the presence of little-explored ecosystem interrelations. We studied the distribution and density of reptiles and mammals in two altitude gradients of $76 \mathrm{~km}$ each. On the other hand, we use a stable isotopes analysis for characterization of the trophic niche in reptiles. We found that the reptiles and mammals are infrequent and have low densities. The silverfish of the Zygentoma order are key prey in this community. Reptiles overlap in the consumption of trophic sources, but differ in their positions in the trophic web. The information is complemented with information about predation among reptiles in this extreme ecosystem.

Keywords: absolute desert, isotopic niche, mammals, reptiles, saurophagy. 


\section{INTRODUCCIÓN}

En Chile, el desierto de Atacama se extiende por $180.000 \mathrm{~km}^{2}$ (Williams et al. 2008) y corresponde a la porción más austral del desierto costero de Perú y Chile, ubicado entre los $5^{\circ}$ y $27^{\circ}$ de latitud Sur. En el área, las condiciones áridas han prevalecido desde el cuaternario tardío (Arroyo et al. 1988). Dentro del desierto de Atacama, se reconoce al desierto interior (Gajardo 1994), el que se extiende entre los $18,35^{\circ}$ y $25,0^{\circ}$ Sur. Corresponde a un área hiperárida (rango anual de precipitaciones entre agosto 2017-2019 de 0 - 3,55 mm, estación meteorológica de Pica, Instituto de Investigaciones Agropecuarias), que carece de vida vegetal, salvo en condiciones muy particulares asociadas a la presencia de aguas subterráneas (bosques de tamarugo y salares).

En este desierto, habitan reptiles como el dragoncito Liolaemus reichei (Werner 1907), especie previamente considerada un sinónimo menor de L. stolzmanni (Steindachner 1891) por Langstroth (2011), pero revalidada por ValladaresFaúndez et al. (2018), y el gecko Phyllodactylus gerrhopygus (Wiegmann 1834). Liolaemus reichei es considerada en Peligro (UICN 2019), corresponde a un lagarto pequeño (SVL 42 $\mathrm{mm}$ ), endémico de Chile, diurno y con distribución restringida (Ruiz de Gamboa \& Ferrú 2013). Phyllodactylus gerrhopygus, está clasificado como Preocupación Menor (UICN 2019), corresponde a un gecko (SVL $50 \mathrm{~mm}$ ), endémico del desierto chileno-peruano y nocturno (Pincheira-Donoso 2006). Dentro de los mamíferos, se describe la presencia del roedor Phyllotis limatus (Thomas 1912), presente en estas áreas desérticas (Lobos \& Alzamora 2016) y como predador, se puede mencionar al zorro Lycalopex culpaeus (Molina 1782) (Guzmán-Sandoval et al. 2007a).

En relación a las especies que habitan en el desierto absoluto de Atacama, los estudios ecológicos se han centrado principalmente en los ambientes más favorables dentro de este ecosistema hiperárido. Así por ejemplo, se pueden mencionar trabajos asociados a Oasis de Nieblas (Sagredo et al. 2002, Guzmán-Sandoval et al. 2007a, Guzmán-Sandoval et al. 2007b) y bosques de la Pampa del Tamarugal (Carmona \& Rivadeneira 2006, Carevic et al. 2013, Ossa et al. 2014, Martínez et al. 2016). Sin embargo, las interacciones que se dan en las condiciones ambientales más adversas (sin vegetación) son prácticamente desconocidas, en especial las de tipo trófica. En este contexto, el uso de isótopos estables (SIA, por su sigla en inglés Stable Isotope Analysis) constituye una herramienta que permite la reconstrucción de la dieta y la determinación de las posiciones tróficas de los organismos (Fry 2006, Davis et al. 2012). Las firmas isotópicas de carbono $\left(\delta^{13} \mathrm{C}\right)$, se han utilizado para identificar las fuentes de producción energética primaria de los consumidores y las del nitrógeno $\left(\delta^{14} \mathrm{~N}\right)$ para estimar el nivel trófico del consumidor (Kupffer et al. 2006, Newsome et al. 2007, Baeta 2019). Dentro de las ventajas del uso de SIA destaca que permiten obtener una visión integrada del consumo que realiza una especie en el tiempo, no requieren necesariamente de un muestreo intensivo en la dimensión temporal y espacial, permiten complementar los estudios dietarios y contribuyen al conocimiento trófico de las comunidades (Grundler et al. 2017); además es considerada una metodología robusta para determinar los alimentos asimilados (Davis et al. 2012). Por otra parte, el uso de SIA tiene un alto potencial en el estudio de especies amenazadas (Molina et al. 2018), permitiendo analizar relaciones tróficas en especies con escaso conocimiento, o donde ellas son difíciles de determinar por los bajos tamaños poblacionales. En reptiles, se reportan escasos estudios en los que se aplica el uso de SIA (Grundler et al. 2017). Para Chile, uno de esos estudios incluyó la utilización de SIA para comparar las dietas de poblaciones insulares y continentales de Liolaemus pictus (Dumeril \& Bibron 1837) (Vidal \& Sabat 2010).

En este contexto, ante la falta de información ecológica de las especies del desierto hiperárido, nuestros objetivos fueron analizar la distribución, densidad y ecología trófica de estas especies. Nuestras predicciones son que las distribuciones de los reptiles y micromamíferos son restringidas (a condiciones de microhábitat que resulten favorables), las densidades son bajas debido a la limitada disponibilidad de recursos y las relaciones tróficas deben ser simples y con una alta sobreposición debido a la restricción de recursos propios de estos sitios hiperáridos.

\section{MATERIALES Y MÉTODOS}

\section{ÁREA DE ESTUDIO}

El estudio se realizó en la región de Tarapacá, norte de Chile, en un área que abarca $2.500 \mathrm{~km}^{2}$. Se caracteriza por la hiperaridez, entendida en un sentido operacional como zonas carentes de cualquier tipo de vegetación (sectores con vegetación azonal fueron descartados de nuestro análisis). Los relevamientos de terreno se realizaron entre el 9 al 29 de abril de 2018.

\section{DISTRIBUCIÓN Y DENSIDAD}

Realizamos dos recorridos longitudinales al norte y sur de la ciudad de lquique (76 km lineales cada uno), entre los 538 y $1.663 \mathrm{msnm}$ (Fig. 1). Se relevaron 47 estaciones de muestreos, en 14 de ellas se muestreo micromamíferos por medio de trampas Sherman (20 trampas por estación de muestreo durante tres noches) e instalación de trampas cámaras durante todo el período; $y$ en todas se realizó transectos para reptiles, 
de 200 metros de largo y 10 de ancho. Para caracterizar los sitios de muestreos, nosotros clasificamos la topología de los sitios como planicie (27 sitios), lomajes (14), quebrada (1) y ladera (5). El sustrato lo clasificamos como arenoso (41 sitios), limoso (4), calcáreo (1), rocoso (1). El grado de intervención antrópica fue desde $0=\sin$ impacto evidente a $4=$ alto impacto a nivel de microhábitat $(0=10$ sitios, $1=21,2=$ $13,3=1,4=2$ ). Adicionalmente, durante un día del periodo de muestreo, instalamos dos datalogger (Tenmars TM-305U), en una guarida de un reptil (sitio N2), un equipo registró la temperatura ambiental afuera y otro adentro de la guarida, por lapsus de una hora. Los transectos fueron inspeccionados en el día (11-16 horas) y al anochecer (19-23 horas).

\section{NicHO ISOTÓPICO}

Nosotros trabajamos específicamente en los sitios TN2; TN-3 y TN-4. Para las potenciales presas, se recolectó invertebrados por medio de dos métodos: (1) búsqueda directa de 1 hora (Molina-Burgos et al. 2018), y (2) con la instalación de 6 trampas de caídas de pvc $(100 \mathrm{~mm}$ diámetro y $130 \mathrm{~mm}$ profundidad), enterradas en el suelo y situadas aproximadamente a 15 metros entre ellas. Cada trampa fue rellenada con $50 \mathrm{ml}$ de agua y jabón durante 3 días consecutivos. Para el análisis de isotopos, se utilizó el cuerpo completo de los invertebrados. A los reptiles se les amputo un trozo de la punta de la cola y a los roedores un pedazo de oreja (4 miligramos), siendo devueltos al medio. Se utilizó $1 \mathrm{mg}$ de tejido de músculo de los vertebrados e igual cantidad del cuerpo de un conjunto representativo de presas. Las muestras fueron molidas, secadas y posteriormente se les extrajo los lípidos con éter de petróleo usando un extractor Soxhlet. Se determinó la composición de isótopos de carbono y nitrógeno $\left(\delta^{13} \mathrm{C}\right.$ y $\left.\delta^{15} \mathrm{~N}\right)$, por medio de un espectrómetro de masas de relación de isótopos de flujo continuo. Se determinó la proporción en que contribuyeron las presas en la dieta de reptiles, debido a la mayor disponibilidad de individuos predadores muestreados, mediante el modelo de mezcla SIAR (Parnell \& Jackson 2013). Los parámetros del modelo fueron estimados ocupando 300.000 iteraciones por medio de cadenas de Markov. Para el factor de enriquecimiento trófico (TEF) se consideró $1,3 \pm 0,3 \delta^{13} \mathrm{C}$ y 2,3 $\pm 0,18 \delta^{15} \mathrm{~N}$ (McCutchan et al. 2003).

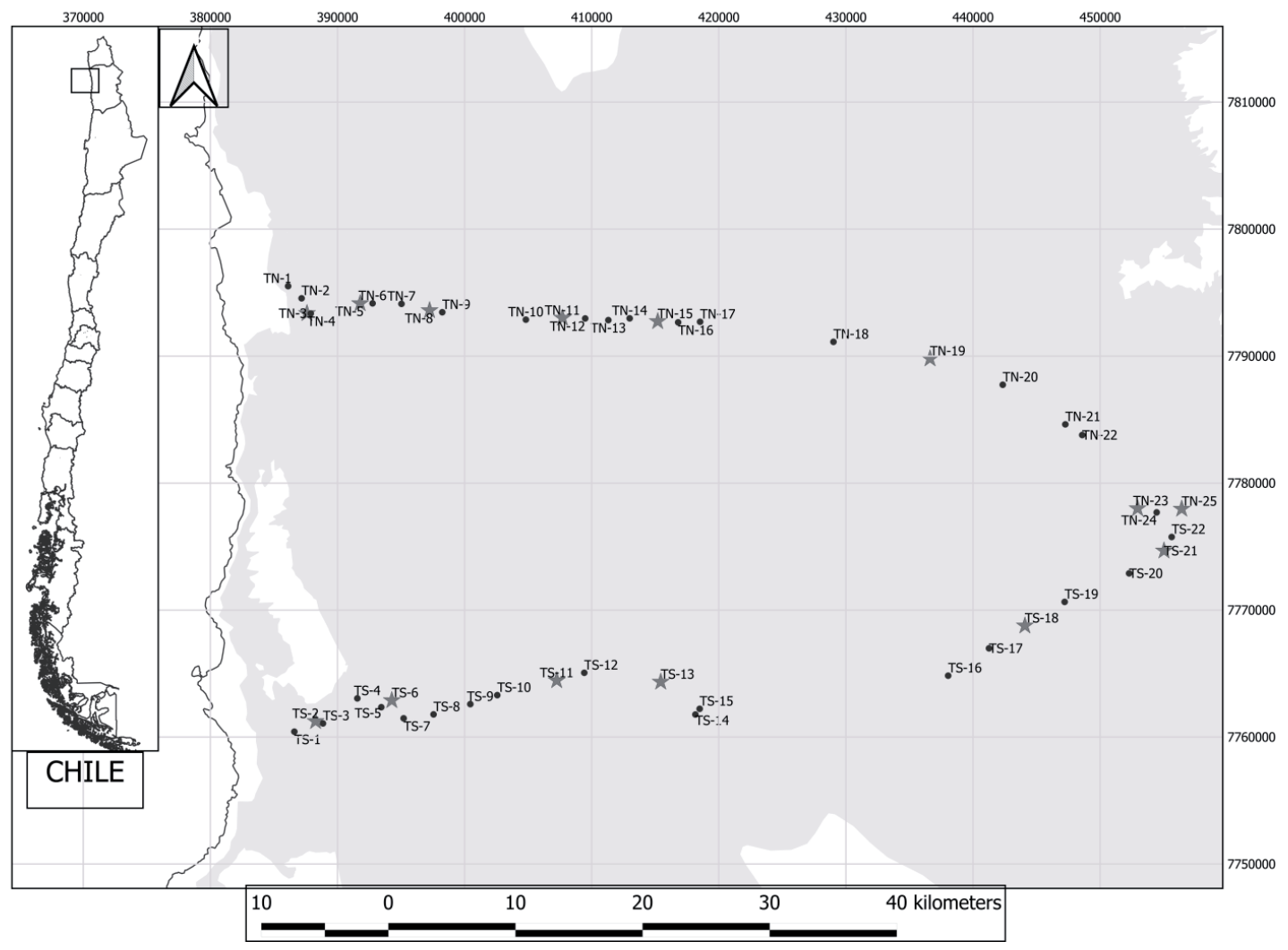

Figura 1. Área de estudio. El desierto interior es representando por el sombreado gris, en color blanco otros ambientes. Los círculos representan sitios donde solo se muestreo reptiles y estrellas donde se incluyó trampas Sherman y trampas cámaras. Coordenadas geográficas son representadas en UTM, datum WGS84, zona 19S. / Study area. The absolute desert is represented by gray shading and other environmental are represent in white color. Circles represent sites where only reptiles were sampled and stars are sites with Sherman traps and camera traps. Geographical coordinates are represented in UTM, datum WGS84, zone 19S. 
Predación entre RePtiles

Adicionalmente reportamos una observación de saurofagia en Liolaemus torresi (Núñez, Navarro, Garín, Pincheira-Donoso \& Meriggio 2003), una especie en Peligro de extinción (UICN 2019), en la localidad de Sierra Gorda ( $22^{\circ} 44^{\prime} \mathrm{S}-69^{\circ} 23^{\prime} \mathrm{W}$ ), en la porción sur del desierto absoluto, región administrativa de Antofagasta.

\section{RESULTADOS}

\section{DISTRIBUCIÓN Y DENSIDAD}

Los reptiles se distribuyeron entre los 640 a 1.663 msnm (Tabla 1, Anexo 1). Phyllodactylus gerrhopygus y L. reichei presentaron predilección por planicies $(45,4$ y $71,4 \%$ ) y loma- jes (36,4 y $28,6 \%$ ), sustrato arenoso (100 y $86 \%$ ) y sitios con bajo grado de perturbación (72,8 y $100 \%$ respectivamente). Ambas especies fueron observadas bajo piedras y guaridas (hoyos), aunque $P$. gerrhopygus también estuvo presente bajo cartones y plásticos. Los dataloggers instalados en una madriguera de L. reichei (Fig. 2), mostraron que durante el día (8:00 a 18:00 horas) las temperaturas fueron más bajas dentro de la guarida (hasta un diferencial de casi $10^{\circ}$ a las 14:00 horas) y levemente mayores durante las 19:00 a 7:00 horas.

De acuerdo a la Tabla 1, los saurios se registraron en un total de 15 sitios (31,9\%). Ambas especies presentaron una baja frecuencia de ocupación, con $P$. gerrhopygus presente en 11 sitios (23,4\%) y L. reichei en 7 (14,89\%) y fueron simpátricos en 3 sitios (6,38\%).

TABla 1. Número de individuos (Ni) y densidad (D) de los reptiles Phyllodactylus gerrhopygus (Phge) y Liolaemus reichei (Lire). Para los mamíferos Canis lupus familiaris (Cafa), Lycalopex culpaeus (Lycu), Mus musculus (Mumu) y Phyllotis limatus (Phli) se entregan datos de presencia y ausencia. Tabla resumida a localidades con registros (total de sitios relevados 47, con 14 sitios para mamíferos). / Number of individuals (Ni) and density (D) of the reptiles Phyllodactylus gerrhopygus (Phge) and Liolaemus reichei (Lire). For mammals Canis lupus familiaris (Cafa), Lycalopex culpaeus (Lycu), Mus musculus (Mumu) and Phyllotis limatus (Phli), presence and absence data are provided. Summary table of localities with records (total of 47 surveyed sites, with 14 sites for mammals).

\begin{tabular}{|c|c|c|c|c|c|c|c|c|c|c|c|}
\hline \multirow[b]{2}{*}{ Sitios } & \multicolumn{2}{|c|}{ Coordenadas } & \multirow{2}{*}{$\frac{\text { Altitud }}{\text { (metros) }}$} & \multicolumn{2}{|c|}{ Phge } & \multicolumn{2}{|c|}{ Lire } & Cafa & Lycu & Mumu & Phli \\
\hline & Oeste & Norte & & $\mathrm{Ni}$ & $\mathrm{D}$ & $\mathrm{Ni}$ & D & \multicolumn{4}{|c|}{ Presente 1/Ausente 0} \\
\hline $\mathrm{TN}-2$ & 387.174 & 7.794 .549 & 661 & 0 & 0 & 1 & 0,05 & - & - & - & - \\
\hline TN-3 & 387.596 & 7.793 .364 & 646 & 1 & 0,05 & 1 & 0,05 & 0 & 0 & 1 & 1 \\
\hline TN-4 & 387.895 & 7.793 .353 & 640 & 1 & 0,05 & 2 & 0,1 & - & - & - & - \\
\hline $\mathrm{TN}-11$ & 407.698 & 7.792 .996 & 1.115 & 1 & 0,05 & 2 & 0,1 & 0 & 1 & 0 & 0 \\
\hline $\mathrm{TN}-12$ & 409.488 & 7.792 .962 & 1.149 & 0 & 0 & 1 & 0,05 & - & - & - & - \\
\hline $\mathrm{TN}-13$ & 411.309 & 7.792 .831 & 1.152 & 0 & 0 & 1 & 0,05 & - & - & - & - \\
\hline TN-15 & 415.193 & 7.792 .730 & 1.116 & 0 & 0 & 0 & 0 & 0 & 1 & 0 & 0 \\
\hline TN-19 & 436.622 & 7.789 .792 & 1.182 & 0 & 0 & 0 & 0 & 0 & 1 & 0 & 0 \\
\hline TN-20 & 442.341 & 7.787 .740 & 1.264 & 1 & 0,05 & 0 & 0 & - & - & - & - \\
\hline $\mathrm{TN}-23$ & 452.951 & 7.777 .992 & 1.538 & 0 & 0 & 0 & 0 & 0 & 1 & 0 & 0 \\
\hline $\mathrm{TN}-24$ & 454.453 & 7.777 .690 & 1.572 & 1 & 0,05 & 0 & 0 & - & - & - & - \\
\hline $\mathrm{TN}-25$ & 456.420 & 7.777 .959 & 1.663 & 1 & 0,05 & 0 & 0 & 0 & 0 & 0 & 0 \\
\hline TS-2 & 388.274 & 7.761 .234 & 659 & 0 & 0 & 0 & 0 & 1 & 0 & 1 & 0 \\
\hline TS-6 & 394.275 & 7.762 .877 & 979 & 1 & 0,05 & 0 & 0 & 0 & 0 & 0 & 0 \\
\hline TS-10 & 402.571 & 7.763 .295 & 1.029 & 1 & 0,05 & 0 & 0 & - & - & - & - \\
\hline TS-11 & 407.248 & 7.764 .450 & 1.096 & 1 & 0,05 & 0 & 0 & 0 & 0 & 0 & 0 \\
\hline TS-13 & 415.429 & 7.764 .334 & 1.051 & 1 & 0,05 & 0 & 0 & 0 & 1 & 1 & 1 \\
\hline TS-15 & 418.496 & 7.762 .220 & 1.030 & 0 & 0 & 1 & 0,05 & - & - & - & - \\
\hline TS-18 & 444.083 & 7.768 .759 & 1.124 & 0 & 0 & 0 & 0 & 0 & 1 & 0 & 0 \\
\hline TS-21 & 455.029 & 7.774.666 & 1.537 & 1 & 0,05 & 0 & 0 & 0 & 0 & 0 & 0 \\
\hline
\end{tabular}


En relación a las abundancias, estas fueron bajas, con densidades de $0,012 \pm 0,02$ ind $/ 100 \mathrm{~m}^{2}$ para $P$. gerrhopygus $y$ $0,010 \pm 0,025 \mathrm{ind} / 100 \mathrm{~m}^{2}$ para L. reichei; nunca sobrepasando de tres animales en los sitios con mayor densidad. En relación a las trampas para micromamíferos, se registró presencia de Mus musculus (Linnaeus 1758) en tres sitios (TS-2, TS-13, TN-3; con captura de un ejemplar en cada sitio) y P. limatus en dos sitios (TN-3, TS-13; un ejemplar en cada sitio). A nivel de predadores, las trampas cámaras revelaron la presencia de Canis lupus familiaris (Linnaeus 1758) en un sitio (TS2), Cathartes aura (Linnaeus 1758), en un sitio (TN-3) y $L$. culpaeus en 6 sitios (TS-13, TS-18, TN-11, TN-15, TN-19, $\mathrm{TN}-23), 43 \%$ del total de sitios con cámaras.

\section{NicHO ISOTÓPICO}

A nivel de presas, solo se obtuvo capturas por medio de búsquedas directas. La comunidad de potenciales presas estuvo conformada por cinco taxa (Tabla 2), destacando Lepisma saccharina (Linnaeus 1758) como el más abundante. Para reptiles, se analizó tres individuos de L. reichei (33\% del total registrado en el área) y 6 P. gerrhopygus (50\%); de este último, se incorporó adicionalmente 3 ejemplares colectados en los alrededores del área muestreada. En roedores solo fue posible incorporar un individuo de cada especie debido a las bajas tasas de capturas (sitios utilizados para el análisis isotópico).
Los valores de carbono $\left(\delta^{13} \mathrm{C}\right)$ y nitrógeno $\left(\delta^{15} \mathrm{~N}\right)$ de presas y predadores son presentados en la Tabla 2. La Figura 3, ilustra el nicho isotópico de la comunidad analizada. Desde el punto de vista del origen de los recursos tróficos, L. reichei consume un subconjunto de los utilizados por $P$. gerrhopygus. Del mismo modo, el isotopo de nitrógeno revela que $P$. gerrhopygus ocupa un nivel trófico mayor a L. reichei, este último, lo comparte con ambos roedores. La posición trófica más alta de la trama, es ocupada por un arácnido del grupo de los sicarios. Los diagramas de mezcla estiman que en $L$. reichei el principal aporte correspondió a Zygentoma (99,84 \pm $0,12)$, luego pseudoescorpiones $(0,11 \pm 0,11)$ y sicarios $(0,05$ $\pm 0,05)$. Para P. gerrhopygus, el orden Zygentoma también representó el principal aporte $(99,3 \pm 0,51)$, seguido por pseudoescorpiones $(0,53 \pm 0,48)$ y sicarios $(0,17 \pm 0,17)$.

\section{SAUROFAGIA}

En agosto de 2016, colectamos dos reptiles disecados, donde observamos muerte por atragantamiento de un Microlophus maminensis (Donoso-Barros 1966), de 180 mm de largo total, al intentar tragar un individuo de $L$. torresi de $100 \mathrm{~mm}$ de largo total (Fig. 4). Los animales fueron depositados en la colección del Museo de Historia Natural y Cultural del Desierto de Atacama, en la ciudad de Calama (MUHNCAL 600-14).

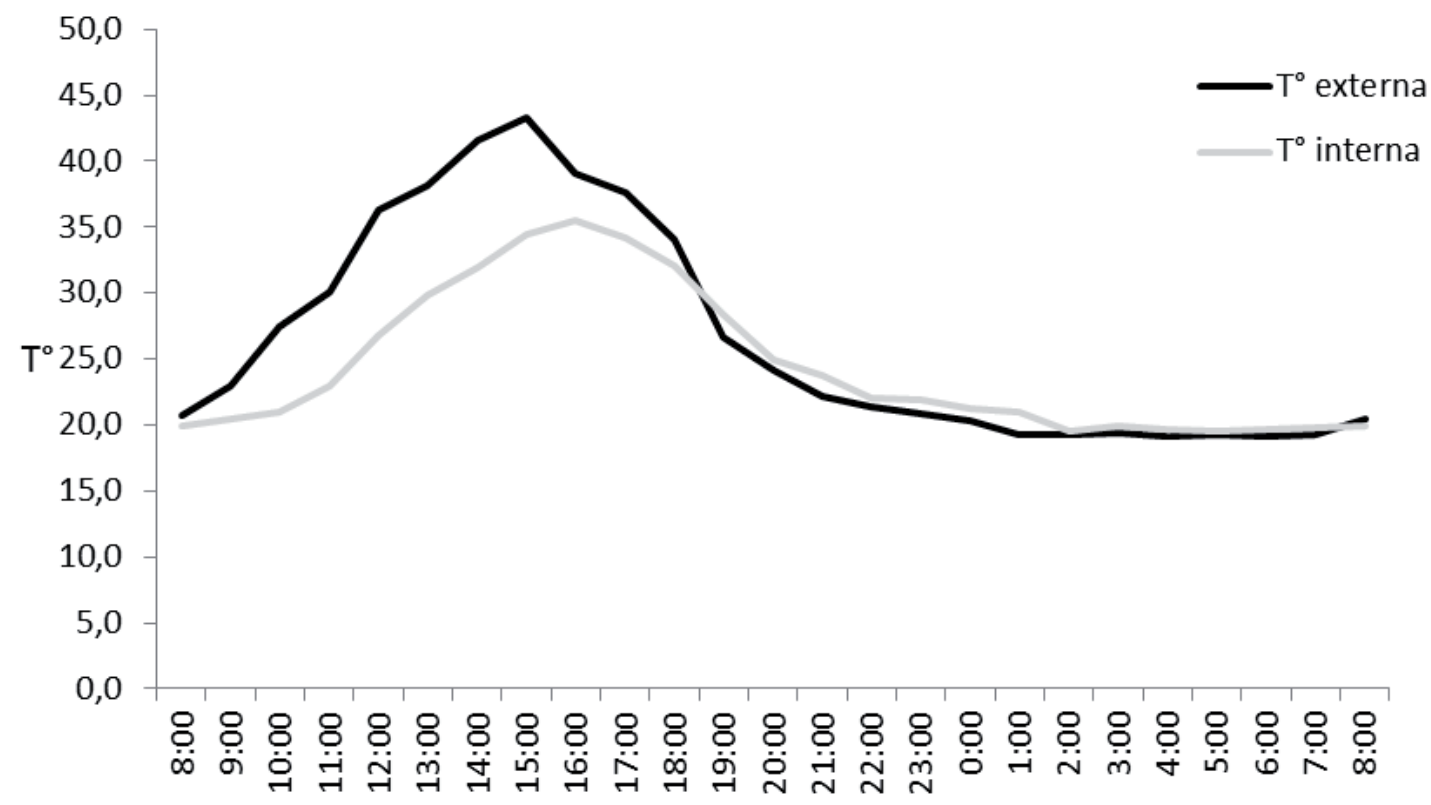

Horas

FIGURA 2. Registros de temperaturas ambientales en una madriguera de L. reichei. Se representan los registros obtenidos en el interior de la madriguera ( $T^{\circ}$ interna) y en el exterior ( $T^{\circ}$ externa). / Records of environmental temperatures in $L$. reichei burrow. The records obtained inside the burrow (internal $\mathrm{T}^{\circ}$ ) and outside (external $\mathrm{T}^{\circ}$ ) are represented. 


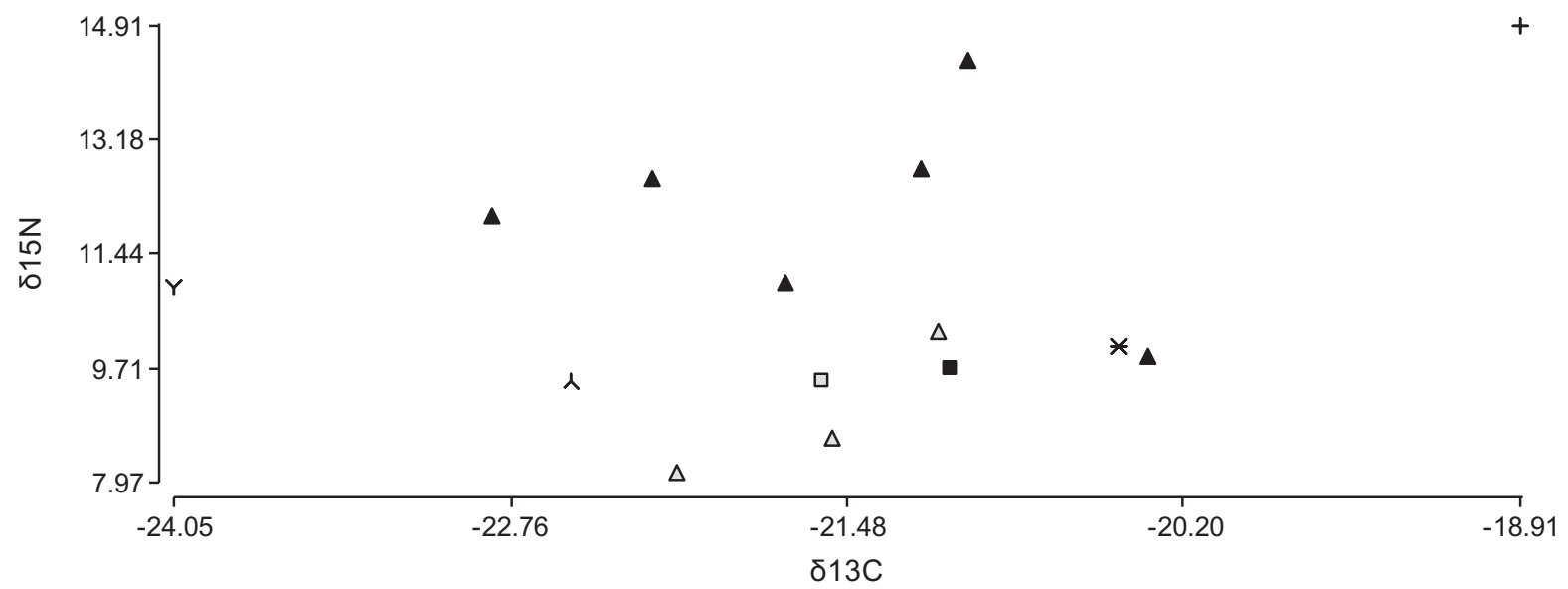

Lesa $\triangle$ Lire $\square$ Mumu $\triangle$ Phge $\square$ Phli $Y$ Pseu + Sica
$\chi$ Tric

Figura 3. Nicho isotópico de una comunidad del desierto hiperárido de Atacama. Lesa Lepisma saccharina, Lire Liolaemus reichei, Mumu Mus musculus, Phge Phyllodactylus gerrhopygus, Phli Phyllotis limatus, Pseu Pseudoescorpionida, Sica Sicarius sp y Tric Tricholepidion sp. / Isotope niche of a community in the hyperarid Atacama Desert. Lesa Lepisma saccharina, Lire Liolaemus reichei, Mumu Mus musculus, Phge Phyllodactylus gerrhopygus, Phli Phyllotis limatus, Pseu Pseudoescorpionida, Sica Sicarius sp. and Tric Tricholepidion sp.

TABLA 2. Comunidad utilizada en estudio de isotopos estables. Ni números de individuos y \%Ni porcentaje del total de individuos. La composición isotópica se expresa como $\left(\delta^{13} \mathrm{C}\right)$ para el carbono, $\left(\delta^{15} \mathrm{~N}\right)$ para el nitrógeno y la DS (desviación estándar) es presentada para los predadores. Las presas fueron agrupadas y analizadas como una muestra compuesta. / Community used in the stable isotope study. $\mathrm{Ni}$ is number of individuals and \% Ni is the percentage of total individuals. The isotope composition is expressed as $\left(\delta^{13} \mathrm{C}\right)$ for carbon, $\left(\delta^{15} \mathrm{~N}\right)$ for nitrogen; SD (standard deviation) is shown for the predators. Prey were grouped and analyzed as a complex sample.

\begin{tabular}{|c|c|c|c|c|c|c|}
\hline Taxa & $\mathrm{Ni}$ & $\% \mathrm{Ni}$ & $\delta^{13} \mathrm{C}$ & $\delta^{15} \mathrm{~N}$ & DS $\delta^{13} \mathrm{C}$ & DS $\delta^{15} \mathrm{~N}$ \\
\hline \multicolumn{7}{|l|}{ Invertebrados } \\
\hline \multicolumn{7}{|l|}{ Aracnida } \\
\hline Sicarius $s p$ & 2 & 11,76 & $-18,9$ & 14,9 & & \\
\hline Pseudoescorpionida & 1 & 5,88 & $-24,1$ & 10,9 & & \\
\hline \multicolumn{7}{|l|}{ Insecta } \\
\hline \multicolumn{7}{|l|}{ Coleoptera } \\
\hline Tenebrionidae & exoesqueletos & - & & & & \\
\hline \multicolumn{7}{|l|}{ Zygentoma } \\
\hline Lepisma saccharina & 13 & 76,47 & $-22,5$ & 9,52 & & \\
\hline Tricholepidion sp & 1 & 5,88 & $-20,4$ & 10 & & \\
\hline \multicolumn{7}{|l|}{ Vertebrados } \\
\hline \multicolumn{7}{|l|}{ Reptilia } \\
\hline Liolaemus reichei & 3 & & $-21,6$ & 8,97 & 0,5 & 1,12 \\
\hline Phyllodactylus gerrhopygus & 6 & & $-21,6$ & 12,1 & 0,9 & 1,55 \\
\hline \multicolumn{7}{|l|}{ Mamíferos } \\
\hline Phyllotis limatus & 1 & & $-21,6$ & 9,51 & & \\
\hline Mus musculus & 1 & & $-21,1$ & 9,71 & & \\
\hline
\end{tabular}




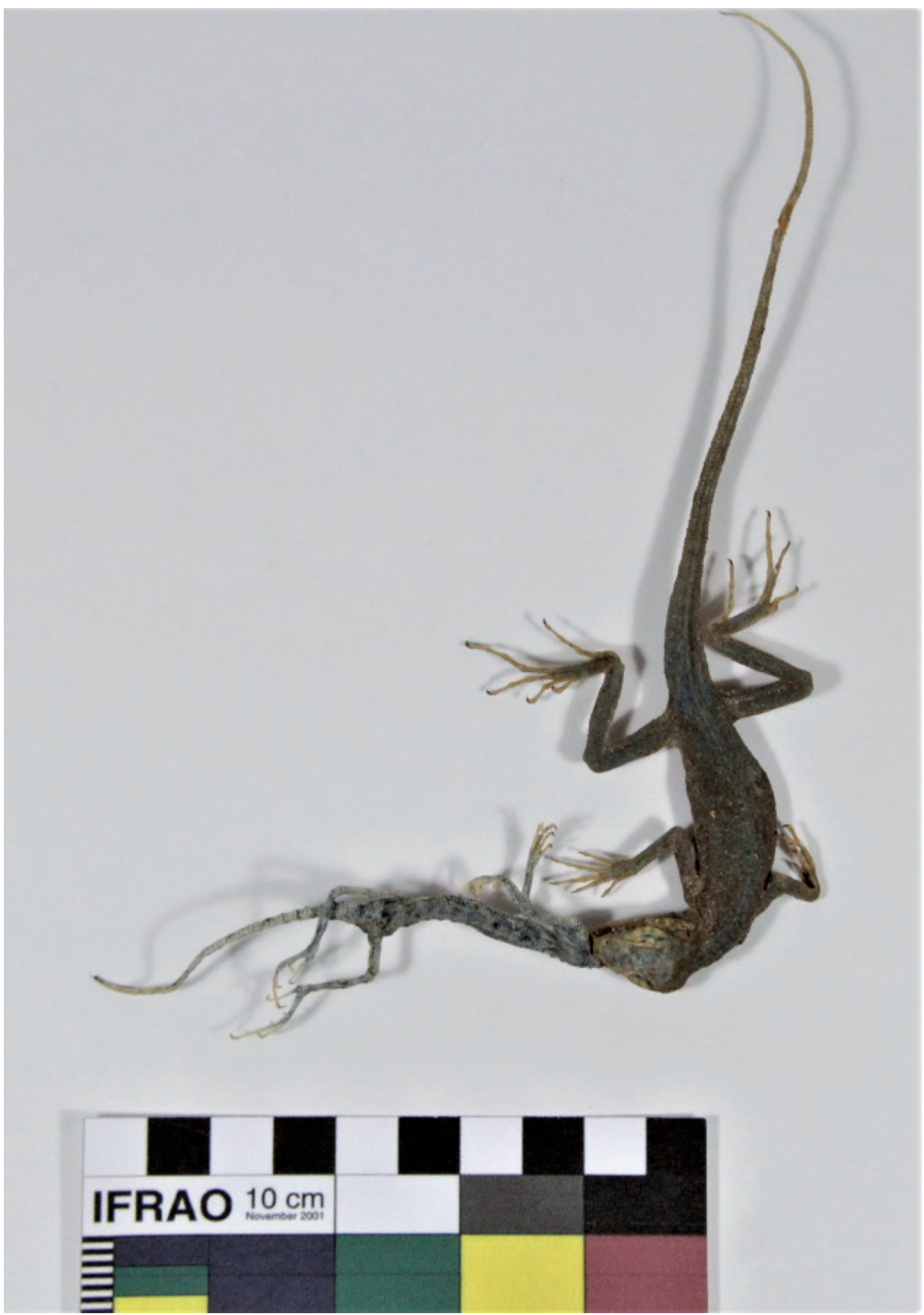

Figura 4. Predación de Microlophus maminensis sobre Liolaemus torresi, Sierra Gorda, Región de Antofagasta. / Predation of Microlophus maminensis on Liolaemus torresi, Sierra Gorda, Región de Antofagasta. 


\section{DISCUSIÓN}

De acuerdo a Noy-Meir (1973), el desierto del norte chileno presenta una productividad que es baja, variable, dependiente de las precipitaciones y de la disponibilidad de nutrientes como el nitrógeno. El área muestreada, se caracterizó por la ausencia de toda forma de vida vegetal, por un predominio de planicies y lomajes, sustrato predominantemente arenoso y por presentar bajo grado de perturbación antrópica a nivel de microhábitat, a diferencia de otras áreas del desierto de Atacama, que presentan un fuerte uso minero y de infraestructura energética. El uso de guaridas bajos piedras (tanto por invertebrados como vertebrados), puede ser relevante para efectos de termorregulación, como se registró en el sitio TN-2. En este sentido, se puede mencionar que en el roedor $P$. limatus, se describe la presencia de guaridas hasta de $1 \mathrm{~m}$ de profundidad en el subsuelo (Lobos \& Alzamora 2016).

En términos poblacionales, los saurios presentaron una distribución asociada a una baja densidad y frecuencia de ocupación. L. reichei, es parte de un conjunto de lagartos conocidos como "dragoncitos", los que son reconocidos por su baja frecuencia de hallazgos (Donoso-Barros 1969). Pese a las condiciones adversas de este ecosistema, hubo registros de roedores, los que fueron escasos. En este sentido, destacó la presencia del cosmopolita M. musculus y su capacidad para invadir este ambiente desértico, siendo a nuestro entender, el primer registro de esta especie para este tipo de ecosistema; es interesante mencionar que esta especie se relaciona a la presencia de habitaciones humanas, las que no están presentes en el área de estudio al menos en la actualidad (en las proximidades del área de estudio, se encontraba el puerto salitrero de Caleta Buena, el que operó entre los años 1888 a 1940). El zorro culpeo, fue registrado en gran parte del área de estudio, favorecido por su mayor capacidad de desplazamiento dentro de este ambiente hostil.

$L$ La red trófica (isotópica) es simple, con $L$. reichei ocupando un rango más estrecho de recursos que $P$. gerrhopygus. Lo anterior, supone una alta competencia entre ambas especies, segregadas por el carácter nocturno del gecko (Pérez \& Balta 2007) y la baja frecuencia en que ambos se superponen. En P. gerrhopygus se reporta en Perú, en base a 5 ejemplares, la presencia de 7 ítems (identificados), donde de acuerdo a un índice de importancia relativa, predominó en primer lugar las arañas, seguido por coleópteros, larvas de insectos y sicarios (Pérez \& Balta 2011). En el estudio mencionado, los individuos fueron registrados en ambientes de desierto, lomas y oasis (los dos últimos desiertos con vegetación).

En relación a la posición trófica, $P$. gerrhopygus ocupa una posición superior a L. reichei. En este sentido, Pérez \& Balta
(2011), destacan el importante consumo de arácnidos por este gecko. Otro aspecto interesante, es la presencia de roedores en un ambiente sin vegetación, que de acuerdo a nuestra red trófica comparten recursos con los reptiles (lo que podría sugerir algún grado de competencia considerando la baja disponibilidad de recursos tróficos), aunque $P$. gerrhopygus consume algunos recursos que le permiten estar en la parte alta de la red trófica. En la red trófica ya mencionada, no se incluyó al zorro culpeo, el que está ampliamente distribuido en el desierto absoluto y que, de acuerdo a estudios previos, es predador de parte de los reptiles de este estudio (Guzmán et al. 2007a).

Los diagramas de mezcla muestran el rol clave de Zygentoma en las dietas de ambos reptiles $(99,8 \%$ para $L$. reichei y $99,3 \%$ para P. gerrhopygus). En este sentido, Lepisma saccharina, fue un invertebrado clave, siendo la presa más abundante. Diversos estudios han explorado la alta capacidad adaptativa de Lepisma, destacando su tolerancia a periodos de inanición extrema (Devries \& Appel 2013) y al hecho de que pueden consumir diversas fuentes de alimentación (Sweetman 1939). Respecto a los otros ítems, destacó que el gecko incorporó 4,8 veces más pseudoescorpiones y 3,4 veces más sicarios que $L$. reichei; ello influyó en su posición trófica. La información de SIA en reptiles, es representativa del uso temporal de los recursos que hacen estos animales, debido a su baja tasa de recambio del nitrógeno producto su carácter poiquilotermo (Grundler et al. 2017), que en el caso de tejidos musculares, puede reflejar una integración de información representativa de semanas a meses (Fisk et al. 2009).

Desde el punto de vista trófico, las relaciones entre especies fueron simples y en algunos casos, pueden ser incluso bidireccionales; por ejemplo, el gecko incorpora arácnidos, aunque existen estudios que describen la predación de arácnidos sobre gekos (Fulvio-Pérez \& Minoli 2014). Este podría ser el caso de la araña Sicarius sp, que ocupo la posición trófica más alta en nuestro estudio. Otro aspecto interesante, es la predación que grandes reptiles como $M$. maminensis pueden hacer sobre reptiles más pequeños. En el caso que reportamos, individuos de L. torresi (dragoncito) fueron observados en los sectores más áridos, próximos a una pequeña quebrada con vegetación (200 metros) ocupada por Microlophus. En este contexto, en el desierto más árido, la falta de recursos tróficos limita la presencia de grandes lagartos, los que están presentes en las áreas arbustivas aledañas al desierto absoluto, lo que podría disminuir el riesgo de saurofagia. Por otra parte, este registro de predación de $M$. maminensis sobre $L$. reichei es el primer reporte de predación para estas especies.

Para los vertebrados del desierto absoluto, hay escasos 
antecedentes de su ecología. Así, nuestros registros van en la dirección de una mejor comprensión de las relaciones ecológicas de las especies que habitan en ambientes extremos como el desierto del sur de Sudamérica.

\section{AGRADECIMIENTOS}

Los autores agradecen el apoyo del Museo de Historia Natural y Cultural del Desierto de Atacama, Calama, Chile. Del mismo modo agradecemos a los revisores de este escrito por sus aportes e importantes sugerencias.

\section{REFERENCIAS}

Arroyo, M.T.K., Squeo, F., Armesto, J.J., Villagrán, C. 1988. Effects of aridity on plant diversity on the northern Chilean Andes: Results of a natural experiment. Annals of the Missouri Botanical Garden 75: 55-78. DOI: $10.2307 / 2399466$

Baeta, A. 2019. Stable Isotope Ecology. En: Fath, B. (Ed) Earth Systems and Environmental Science, Encyclopedia of Ecology: 606-615. Elsevier Reference Collection. DOI: 10.1016/B978-0-12-409548-9.10915-7

Carevic, F., Carmona, E., Muñoz-Pedreros, A. 2013. Seasonal diet of the burrowing owl Athene cunicularia Molina, 1782 (Strigidae) in a hyperarid ecosystem of the Atacama Desert in northern Chile. Journal of Arid Environments 97: 237-241. DOI: 10.1016/j.jaridenv.2013.07.008

Carmona, E., Rivadeneira, M. 2006. Food habitats of the barn owl Tyto alba in the National Reserve Pampa del Tamarugal, Atacama Desert, north Chile. Journal of Natural History (7-8): 473-483. DOI: 10.1080/00222930600699904

Davis, A., Blanchette, M., Pusey, B., Jardine, T., Pearson, R. 2012. Gut content and stable isotope analyses provide complementary understanding of ontogenetic dietary shifts and trophic relationships among fishes in a tropical river. Freshwater Biology 57: 2056-2072. DOI: 10.1111/j.1365-2427.2012.02858.x

Devries, Z.C., Appel, A.G. 2013. Standard metabolic rates of Lepisma saccharina and Thermobia domestica: Effects of temperature and mass. Journal of Insect Physiology, 59(6): 638-645. DOI: 10.1016/j.jinsphys.2013.04.002

Donoso-Barros, R. 1969. Observaciones in vivo sobre Phrynosaura reichei Werner (Sauria-Iguanidae). Boletín de la Sociedad de Biología de Concepción 41: 85-87.

Fisk, A., Sash, K., Maerz, J., Palmer, W., Carroll, J.P., MacNeil, M.A. 2009. Metabolic turnover rates of carbon and nitrogen stable isotopes in captive juvenile snakes. Rapid communication in Mass Spectrometry 23: 319-326. DOI: 10.1002/rcm.3880

Fry, B. 2006. Stable Isotope Ecology. Springer Science and Business Media, LLC. 233. New York, United States. $182 \mathrm{pp}$.
Fulvio-Pérez, H., Minoli, I. 2014. Depredación de Homonota darwinii Laurent 1984 (Squamata: Phyllodactylidae) por Brothriurus burmeisteri Kraepelin 1894 (Scorpiones: Brothriuridae) en la provincia del Chubut, Argentina. Cuadernos de Herpetología 28(2): 145-146.

Gajardo, R., 1994. La Vegetación Natural de Chile: clasificación y distribución geográfica. Editorial Universitaria. Santiago, Chile. 165 pp.

Grundler, MR., Pianka, ER., Pelegrin, N., Cowan, MA., Rabosky DL. 2017. Stable isotope ecology of a hyper-diverse community of scincid lizards from arid Australia. PLoS ONE 12(2): e0172879. DOI: 10.1371/journal. pone.0172879

Guzmán-Sandoval, J., Sielfed, W., Ferru, M. 2007a. Dieta de Lycalopex culpaeus (Mammalia: Canidae) en el extremo norte de Chile (Región de Tarapaca). Gayana 71(1): 1-7.

Guzmán-Sandoval, J., Ferru, M., Ruiz de Gamboa, M., Sielfed, W. 2007b. Artrópodos y vertebrados de un Tillandsial al interior Iquique, norte de Chile, con un listado de taxa previamente conocidos. Boletín de la Sociedad de Biología de Concepción 78: 23-34.

Kupfer, A., Langel, R., Scheu, S., Himstedt, W., Maraun, M. 2006. Trophic ecology of a tropical aquatic and terrestrial food web: insights from stable isotopes (815 N). Journal of Tropical Ecology 22: 469-476. DOI: 10.1017/ S0266467406003336

Langstroth, R.P. 2011. On the species identities of a complex Liolaemus fauna from the Altiplano and Atacama Desert: insights on Liolaemus stolzmanni, L. reichei, L. jamesi pachecoi, and L. poconchilensis (Squamata: Liolaemidae). Zootaxa 2809: 20-32. DOI: 10.11646/zootaxa.2809.1.2

Lobos, G., Alzamora, A. 2016. Densidad y desplazamiento de Phyllotis limatus, un roedor del desierto de Atacama. Gayana 80(1): 56-60.

Martínez, J., Vásquez, R., Marqués A., Díez-Fernández, A., Merino, S. 2016. The prevalence and molecular characterization of blood parasites infecting the vulnerable Tamarugo Conebill (Conirostrum tamarugense) and other birds in the Pampa del Tamarugal, Chile. Emu - Austral Ornithology 116(3): 310-314. DOI: 10.1071/ MU15090

McCutchan, J.H., Lewis, W.M., Kendall, C., Mcgrath, C.C. 2003. Variation in trophic shift for stable isotope ratios of carbon, nitrogen, and sulfur. Oikos 102: 378-390. DOI: 10.1034/j.1600-0706.2003.12098.x

Molina-Burgos, B., Valenzuela, A., Alvarado, M., Klarian, S., Soto, C. 2018. Trophic ecology of the Endangered Darwin's frog inferred by stable isotopes. Endangered Species Research 36: 269-278. DOI: 10.3354/esr00906

Newsome, S.D., Martinez del Rio, C., Bearhop, S., Phillips, D.L. 2007. A niche for isotopic ecology. Frontiers in Ecology and the Environment 5(8): 429-436. DOI: 10.1890/060150.1

Noy-Meir, I. 1973. Desert ecosystems I. Environment and producers. Annual Review of Ecology and Systematics 4: 25-52.

Ossa, G., Bonacic, C., Barquez, R. 2014. First record of 
Histiotus laephotis (Thomas, 1916) from Chile and new distributional information for Histiotus montanus (Phillipi and Landbeck, 1861) (Chiroptera, Vespertilionidae). Mammalia 79(4). DOI: 10.1515/mammalia-2014-0041

Parnell, A., Jackson, A. 2013. SIAR: stable isotope analysis in $\mathrm{R}$ (R package version 4.2). URL: http://CRAN.R-project. orgpackage=siar. Accedido: Enero 20, 2019.

Pérez, J., Balta, K., 2007. Ecología de la comunidad de saurios diurnos de la Reserva Nacional de Paracas, Ica, Perú. Revista Peruana de Biología 13(3):169-176.

Pérez, J., Balta, K. 2011. Ecología de Phyllodactylus angustidigitus y P. gerrhopygus (Squamata: Phyllodactylidae) de la Reserva Nacional de Paracas, Perú. Revista Peruana de Biología 18(2): 217-223.

Pincheira-Donoso, D. 2006. Geckos of Chile (Scleroglossa, Gekkonidae, Gekkoninae). Part II. Biogeography and ontogenetic shifts in the colour pattern of Phyllodactylus gerrhopygus. Can the evidence support the presence of Phyllodactylus inaequalis in Chile? Multequina 15: 37-48.

Ruiz de Gamboa, M., Ferrú, M. 2013. Liolaemus stolzmanni (Steindachner, 1891) (Squamata: Liolaemidae): distribution update. Checklist 9(5): 1067-1069. DOI: 10.15560/9.5.1067

Sagredo, E., Larraín, H., Cereceda, P., Ugarte, A., Osses, P., Farías, M. 2002. Variación Espacio-temporal de la Entomofauna de Coleópteros en el Oasis de Niebla de Alto Patache (2049's; 7009'w) y su relación con factores geográficos. Revista de Geografía Norte Grande (29): 121-133.

Sweetman, H.L. 1939. Responses of the Silverfish, Lepisma saccharina L., to Its Physical Environment*. Journal of Economic Entomology 32(5): 698-700.

Valladares-Faúndez, P., Etheridge, R., Simón-Abdala, C. 2018. Resurrection and redescription of Liolaemus reichei, proposal of a neotype to stabilize its taxonomy. Revista Mexicana de Biodiversidad 89: 393-401. DOI: 10.22201/ ib.20078706e.2018.2.2086

Vidal, MA., Sabat, P. 2010. Stable isotopes document mainlandisland divergence in resource use without concomitant physiological changes in the lizard Liolaemus pictus. Comparative Biochemistry and Physiology, Part B 156: 61-67. DOI: 10.1016/j.cbpb.2010.02.005

UICN. 2019. The UICN Red List of Threatened Species. Version 2019-2. URL: https://www.iucnredlist.org. Accedido: Octubre 21, 2019.

Williams, A., Santoro, C.M., Smith, M.A., Latorre, C. 2008. The impact of Enso in the Atacama Desert and Australian arid zone: exploratory time-series analysis of archaeological records. Chungara, Revista de Antropología Chilena 40: 245-259. DOI: 10.4067/S0717-73562008000300003

Received: 03.01.2020

Accepted: 15.07.2020

Anexo 1: Número de individuos (Ni) y densidad (D) de los reptiles Phyllodactylus gerrhopygus (Phge) y Liolaemus reichei (Lire). Para los mamíferos Canis lupus familiaris (Cafa), Lycalopex culpaeus (Lycu), Mus musculus (Mumu) y Phyllotis limatus (Phli) se entregan datos de presencia y ausencia. / Number of individuals (Ni) and density (D) of the reptiles Phyllodactylus gerrhopygus (Phge) and Liolaemus reichei (Lire). For mammals Canis lupus familiaris (Cafa), Lycalopex culpaeus (Lycu), Mus musculus (Mumu) and Phyllotis limatus (Phli), presence and absence data are provided.

\begin{tabular}{ccccccccccc}
\hline & \multicolumn{2}{c}{ Coordenadas } & Altitud & \multicolumn{2}{c}{ Phge } & Lire & Cafa & Lycu & Mumu & Phli \\
\hline Sitios & Norte & Oeste & (metros) & Ni & D & Ni & D & Presente 1/Ausente 0 & \\
\hline TN-1 & 7.795 .507 & 386.114 & 647 & 0 & 0 & 0 & 0 & & & \\
TN-2 & 7.794 .549 & 387.174 & 661 & 0 & 0 & 1 & 0,05 & & & 1 \\
TN-3 & 7.793 .364 & 387.596 & 646 & 1 & 0,05 & 1 & 0,05 & 0 & 0 & 1 \\
TN-4 & 7.793 .353 & 387.895 & 640 & 1 & 0,05 & 2 & 0,1 & & & \\
TN-5 & 7.794 .140 & 391.781 & 840 & 0 & 0 & 0 & 0 & 0 & 0 & 0 \\
TN-6 & 7.794 .152 & 392.759 & 903 & 0 & 0 & 0 & 0 & & & 0 \\
TN-7 & 7.794 .111 & 395.036 & 1.013 & 0 & 0 & 0 & 0 & & & 0 \\
TN-8 & 7.793 .594 & 397.243 & 1.010 & 0 & 0 & 0 & 0 & 0 & 0 & 0 \\
TN-9 & 7.793 .452 & 398.244 & 1.015 & 0 & 0 & 0 & 0 & & & 0 \\
TN-10 & 7.792 .868 & 404.821 & 1.108 & 0 & 0 & 0 & 0 & & & 0 \\
TN-11 & 7.792 .996 & 407.698 & 1.115 & 1 & 0,05 & 2 & 0,1 & 0 & 1 & 0 \\
TN-12 & 7.792 .962 & 409.488 & 1.149 & 0 & 0 & 1 & 0,05 & & & 0 \\
\hline
\end{tabular}




\begin{tabular}{|c|c|c|c|c|c|c|c|c|c|c|c|c|}
\hline \multirow[b]{2}{*}{ Sitios } & \multicolumn{2}{|c|}{ Coordenadas } & \multirow{2}{*}{$\begin{array}{l}\text { Altitud } \\
\text { (metros) }\end{array}$} & \multicolumn{2}{|c|}{ Phge } & \multicolumn{2}{|c|}{ Lire } & \multirow[t]{2}{*}{ Cafa } & \multicolumn{2}{|l|}{ Lycu } & Mumu & \multirow[t]{2}{*}{ Phli } \\
\hline & Norte & Oeste & & $\mathrm{Ni}$ & D & $\mathrm{Ni}$ & D & & Presente & $1 /$ & Ausente 0 & \\
\hline $\mathrm{TN}-13$ & 7.792 .831 & 411.309 & 1.152 & 0 & 0 & 1 & 0,05 & & & & & \\
\hline TN-14 & 7.792.971 & 412.989 & 1.129 & 0 & 0 & 0 & 0 & & & & & \\
\hline TN-15 & 7.792.730 & 415.193 & 1.116 & 0 & 0 & 0 & 0 & ( & 0 & 1 & 0 & 0 \\
\hline TN-16 & 7.792 .657 & 416.802 & 1.109 & 0 & 0 & 0 & 0 & & & & & \\
\hline $\mathrm{TN}-17$ & 7.792 .699 & 418.530 & 1.115 & 0 & 0 & 0 & 0 & & & & & \\
\hline TN-18 & 7.791.121 & 429.020 & 1.105 & 0 & 0 & 0 & 0 & & & & & \\
\hline TN-19 & 7.789.792 & 436.622 & 1.182 & 0 & 0 & 0 & 0 & ( & 0 & 1 & 0 & 0 \\
\hline $\mathrm{TN}-20$ & 7.787.740 & 442.341 & 1.264 & 1 & 0,05 & 0 & 0 & & & & & \\
\hline TN-21 & 7.784 .622 & 447.264 & 1.360 & 0 & 0 & 0 & 0 & & & & & \\
\hline TN-22 & 7.783.783 & 448.591 & 1.426 & 0 & 0 & 0 & 0 & & & & & \\
\hline $\mathrm{TN}-23$ & 7.777.992 & 452.951 & 1.538 & 0 & 0 & 0 & 0 & ( & 0 & 1 & 0 & 0 \\
\hline TN-24 & 7.777 .690 & 454.453 & 1.572 & 1 & 0,05 & 0 & 0 & & & & & \\
\hline $\mathrm{TN}-25$ & 7.777.959 & 456.420 & 1.663 & 1 & 0,05 & 0 & 0 & ( & 0 & 0 & 0 & 0 \\
\hline TS-1 & 7.760 .420 & 386.604 & 538 & 0 & 0 & 0 & 0 & & & & & \\
\hline TS-2 & 388.274 & 7.761 .234 & 659 & 0 & 0 & 0 & 0 & 1 & 1 & 0 & 1 & 0 \\
\hline TS-3 & 7.761 .069 & 388.858 & 657 & 0 & 0 & 0 & 0 & & & & & \\
\hline TS-4 & 7.763 .041 & 391.560 & 866 & 0 & 0 & 0 & 0 & & & & & \\
\hline TS-5 & 7.762 .347 & 393.446 & 951 & 0 & 0 & 0 & 0 & & & & & \\
\hline TS-6 & 7.762 .877 & 394.275 & 979 & 1 & 0,05 & 0 & 0 & c & 0 & 0 & 0 & 0 \\
\hline TS-7 & 7.761.471 & 395.205 & 903 & 0 & 0 & 0 & 0 & & & & & \\
\hline TS-8 & 7.761 .780 & 397.556 & 875 & 0 & 0 & 0 & 0 & & & & & \\
\hline TS-9 & 7.762 .588 & 400.450 & 914 & 0 & 0 & 0 & 0 & & & & & \\
\hline TS-10 & 7.763 .295 & 402.571 & 1.029 & 1 & 0,05 & 0 & 0 & & & & & \\
\hline TS-11 & 7.764 .450 & 407.248 & 1.096 & 1 & 0,05 & 0 & 0 & c & 0 & 0 & 0 & 0 \\
\hline TS-12 & 7.765 .058 & 409.416 & 1.089 & 0 & 0 & 0 & 0 & & & & & \\
\hline TS-13 & 7.764 .334 & 415.429 & 1.051 & 1 & 0,05 & 0 & 0 & c & 0 & 1 & 1 & 1 \\
\hline TS-14 & 7.761.772 & 418.159 & 1.032 & 0 & 0 & 0 & 0 & & & & & \\
\hline TS-15 & 7.762 .220 & 418.496 & 1.030 & 0 & 0 & 1 & 0,05 & & & & & \\
\hline TS-16 & 7.764 .830 & 438.045 & 1.050 & 0 & 0 & 0 & 0 & & & & & \\
\hline TS-17 & 7.766 .984 & 441.260 & 1.062 & 0 & 0 & 0 & 0 & & & & & \\
\hline TS-18 & 444.083 & 7.768.759 & 1.124 & 0 & 0 & 0 & 0 & c & 0 & 1 & 0 & 0 \\
\hline TS-19 & 7.770 .640 & 447.210 & 1.236 & 0 & 0 & 0 & 0 & & & & & \\
\hline TS-20 & 7.772 .888 & 452.281 & 1.416 & 0 & 0 & 0 & 0 & & & & & \\
\hline TS-21 & 7.774 .666 & 455.029 & 1.537 & 1 & 0,05 & 0 & 0 & ( & 0 & 0 & 0 & 0 \\
\hline TS-22 & 7.775 .753 & 455.633 & 1.587 & 0 & 0 & 0 & 0 & & & & & \\
\hline
\end{tabular}

